\title{
Hook Length Polynomials for Plane Forests of a Certain Type
}

Fu Liu

Department of Mathematics, University of California, Davis, One Shields Avenue, Davis, CA 95616, USA

fuliu@math.ucdavis.edu

Received January 21, 2007

AMS Subject Classification: 05A15, 05A19

\begin{abstract}
The original motivation for the study of hook length polynomials was to find a combinatorial proof for a hook length formula for binary trees given by Postnikov, as well as a proof for a hook length polynomial formula conjectured by Lascoux. In this paper, we define the hook length polynomial for plane forests of a given degree sequence type and show that it can be factored into a product of linear forms. Some other enumerative results on forests are also given.
\end{abstract}

Keywords: hook length, plane forest

\section{Introduction}

In [3], Du and the author defined the hook length polynomials for $m$-ary trees and showed that they can be written as simple binomial expressions. In this paper, we extend this result to plane forests of a given degree sequence type.

The original motivation for the work on hook length formulas was to seek a combinatorial proof of an identity derived by Postnikov [7, 8]:

$$
\sum_{T} \frac{n !}{2^{n}} \prod_{v}\left(1+\frac{1}{h_{v}}\right)=(n+1)^{n-1},
$$

where the sum is over all complete binary trees with $n$ internal vertices, the product is over all internal vertices of $T$, and $h_{v}$ is the "hook length" of $v$ in $T$, namely, the number of internal vertices in the subtree of $T$ rooted at $v$.

Chen and Yang [2] and Seo [9] both gave direct bijective proofs for (1.1). Moreover, based on (1.1), Lascoux replaced 1 with $x$ and conjectured a hook length polynomial formula for binary trees:

$$
\sum_{T} \prod_{v}\left(x+\frac{1}{h_{v}}\right)=\frac{1}{(n+1) !} \prod_{i=0}^{n-1}((n+1+i) x+n+1-i) .
$$


Du and the author [3] generalized Lascoux's conjecture and proved hook length polynomial formulas for $m$-ary trees and plane forests. Analogous results were also given by Gessel and Seo [5].

In Section 2, we define hook length polynomial for forests corresponding to a given degree sequence and show that it has a simple binomial form. In Section 3, we study another form of hook length polynomials (3.2) and get an enumerative result on colored labelled forests (3.4) by using the idea of proper vertices introduced by Seo [9]. These techniques allow a fully bijective proof of (3.4), which then yields new fully bijective proofs for the formulas (2.1) and (2.2).

\section{Hook Length Polynomials for Plane Forests of Type $\mathbf{r}$}

A tree is an acyclic connected graph. For any vertices $v$ and $u$ in a tree, we call $v$ a descendant of $u$ (or $u$ an ancestor of $v$ ) if $u$ lies on the unique path from the root to $v$. In particular, if $u$ and $v$ are adjacent, we call $v$ a child of $u$. For any vertex $v$, we use $\operatorname{Des}(v)$ to denote the set of descendants of $v$.

For any vertex $v$ in a tree, the degree of $v$ is the number of children of $v$. A vertex is an internal vertex if it is not a leaf, i.e., its degree is not zero. A plane tree is an unlabelled rooted tree whose vertices are regarded as indistinguishable, but the subtrees at any vertex are linearly ordered. A plane forest is a finite set of ordered plane trees. For any plane forest $F$, we denote by $I(F)$ the set of internal vertices of $F$.

For any plane forest $F$, let $r_{i}$ be the number of vertices of degree $i$ and $\mathbf{r}=$ $\left(r_{0}, r_{1}, r_{2}, \ldots\right)$, then we say that $F$ is of type $\mathbf{r}$. Given a nonnegative integer sequence $\mathbf{r}=\left(r_{0}, r_{1}, r_{2}, \ldots\right)$ with $\sum_{d \geq 0} r_{d}<\infty$, we use $\mathscr{F}(\mathbf{r})$ to denote all the forests $F$ of type r.

There is a well-known result on the cardinality of $\mathscr{F}(\mathbf{r})[1,4,6,10]$, denoting by $n=\sum_{d \geq 1} r_{d}=|I(F)|$ the number of internal vertices and $\ell=-\sum_{d \geq 0}(d-1) r_{d}$ the number of trees in $F$ :

$$
|\mathscr{F}(\mathbf{r})|=\frac{\ell}{n+r_{0}}\left(\begin{array}{c}
n+r_{0} \\
r_{0}, r_{1}, r_{2}, \ldots
\end{array}\right) .
$$

Definition 2.1. For any vertex $v$ of a forest $F$, let $d_{v}$ be its degree and $h_{v}$ its hook length, i.e., the number of descendants it has. We define the hook length polynomial of $v$ as

$$
P_{v}(x)=\frac{\left(\left(d_{v}-1\right) h_{v}+1\right) x+1-h_{v}}{d_{v} h_{v}} .
$$

Definition 2.2. We define the hook length polynomial for plane forests of type $\mathbf{r}$ as

$$
\mathcal{H}_{\mathbf{r}}(x)=\sum_{F \in \mathscr{F}(\mathbf{r})} \prod_{v \in I(F)} P_{v}(x) .
$$

Then $\mathcal{H}_{\mathbf{r}}(x)$ can be written as a binomial expression:

Theorem 2.3.

$$
\mathcal{H}_{\mathbf{r}}(x)=\frac{\ell}{r_{0}}\left(\begin{array}{c}
r_{0} x \\
r_{0} x-n, r_{1}, r_{2}, \ldots
\end{array}\right) .
$$


Proof. If we replace $x$ by $k$, then the right side of (2.2) becomes

$$
\frac{\ell}{r_{0}}\left(\begin{array}{c}
k r_{0} \\
k r_{0}-n, r_{1}, r_{2}, \ldots
\end{array}\right)=\frac{k \ell}{k r_{0}}\left(\begin{array}{c}
k r_{0} \\
k r_{0}-n+r_{1}, r_{2}, r_{3}, \ldots
\end{array}\right)\left(\begin{array}{c}
k r_{0}-n+r_{1} \\
r_{1}
\end{array}\right) .
$$

Applying (2.1), one can see that it counts the number of plane forests of type $\mathbf{r}^{\prime}=\left(r_{0}^{\prime}, r_{1}^{\prime}, r_{2}^{\prime}, \ldots\right)$, with $r_{1}$ leaves circled, where $r_{0}^{\prime}=k r_{0}-n+r_{1}, r_{i k}^{\prime}=r_{i+1}, \forall i \geq 1$ and $r_{j}^{\prime}=0$ for all $j \neq i k$ for any $i$. (Note that $\left(k r_{0}-n+r_{1}\right)+\sum_{d \geq 2} r_{d}=k r_{0}$ and $\left(k r_{0}-n+r_{1}\right)-\sum_{d \geq 2}((d-1) k-1) r_{d}=k \ell$. $)$

Because both sides of (2.2) are polynomials in $x$, it's enough to prove that

$$
\mathcal{H}_{\mathbf{r}}(k)=\frac{\ell}{r_{0}}\left(\begin{array}{c}
r_{0} k \\
r_{0} k-n, r_{1}, r_{2}, \ldots
\end{array}\right) \text {. }
$$

We prove this by induction on $n$, the number of internal vertices of $F$.

When $n=0$, we have $\mathbf{r}=\left(r_{0}, 0,0, \ldots\right)$ and $\ell=r_{0}$, so

$$
\mathcal{H}_{\mathbf{r}}(k)=1=\frac{\ell}{r_{0}}\left(\begin{array}{c}
r_{0} k \\
r_{0} k
\end{array}\right) .
$$

Assume (2.3) holds for $n<n_{0}$. Now we consider $n=n_{0}$.

If $\ell=1$, then $\forall F \in \mathscr{F}(\mathbf{r}), F$ is just a tree, say, $T$. Let $v_{0}$ be the root of $T$. Then

$$
\mathcal{H}_{\mathbf{r}}(k)=\sum_{d \geq 1, r_{d} \neq 0} \frac{((d-1) n+1) k+1-n}{d n} \mathcal{H}_{\mathbf{r}(d)}(k),
$$

where $\mathbf{r}^{(d)}=\left(r_{0}, r_{1}, \ldots, r_{d-1}, r_{d}-1, r_{d+1}, \ldots\right)$.

By the induction hypothesis,

$$
\begin{aligned}
\mathcal{H}_{\mathbf{r}^{(d)}}(k) & =\frac{d}{r_{0}}\left(\begin{array}{c}
r_{0} k \\
r_{0} k-n+1, r_{1}, \ldots, r_{d-1}, r_{d}-1, r_{d+1}, \ldots
\end{array}\right) \\
& =\frac{d}{r_{0}} \frac{r_{d}}{r_{0} k-n+1}\left(\begin{array}{c}
r_{0} k \\
r_{0} k-n, r_{1}, \ldots, r_{d-1}, r_{d}, r_{d+1}, \ldots
\end{array}\right) .
\end{aligned}
$$

Therefore,

$$
\begin{aligned}
\mathcal{H}_{\mathbf{r}}(k) & =\sum_{d \geq 1, r_{d} \neq 0} \frac{((d-1) n+1) k+1-n}{r_{0} n} \frac{r_{d}}{r_{0} k-n+1}\left(\begin{array}{c}
r_{0} k \\
r_{0} k-n, r_{1}, r_{2}, \ldots
\end{array}\right) \\
& =\frac{1}{r_{0}}\left(\begin{array}{c}
r_{0} k \\
r_{0} k-n, r_{1}, r_{2}, \ldots
\end{array}\right) \sum_{d \geq 1, r_{d} \neq 0} \frac{(((d-1) n+1) k+1-n) r_{d}}{n\left(r_{0} k-n+1\right)} \\
& =\frac{1}{r_{0}}\left(\begin{array}{c}
r_{0} k \\
r_{0} k-n, r_{1}, r_{2}, \ldots
\end{array}\right) .
\end{aligned}
$$


If $\ell>1$,

$$
\begin{aligned}
\mathcal{H}_{\mathbf{r}}(k) & =\sum_{\mathbf{r}=\mathbf{r}^{(1)}+\mathbf{r}^{(2)}+\cdots+\mathbf{r}^{(\ell)}} \prod_{i=1}^{\ell} \mathcal{H}_{\mathbf{r}^{(i)}}(k) \\
& =\sum_{\mathbf{r}=\mathbf{r}^{(1)}+\mathbf{r}^{(2)}+\cdots+\mathbf{r}^{(\ell)}} \prod_{i=1}^{\ell} \# \text { of forests of type } \mathbf{r}^{(i)^{\prime}} \text { with } \mathbf{r}_{1}^{(i)} \text { leaves circled } \\
& =\# \text { of forests of type } r^{\prime} \text { with } r_{1} \text { leaves circled } \\
& =\frac{\ell}{r_{0}}\left(\begin{array}{c}
r_{0} k \\
r_{0} k-n, r_{1}, r_{2}, \ldots
\end{array}\right) .
\end{aligned}
$$

\section{Colored Labelled Forests}

In this section, we will consider labelled plane forests. Given a plane forest $F$ with $n$ internal vertices, a labelling is a bijection from $I(F)$ to $[n]$. A labelled forest is a plane forest with a labelling. For a vertex $v$ in a labelled forest, following [9], we call $v$ a proper vertex if none of its descendants has smaller label than $v$, and an improper vertex otherwise. For a labelled forest $F$, we use $\operatorname{Prop}(F)$ to denote the set of proper vertices and $\operatorname{Improp}(F)$ the set of improper vertices.

Suppose that we have two sets of colors $\left\{c_{1}, c_{2}, \ldots\right\}$ and $\left\{c_{1}^{\prime}, c_{2}^{\prime}, \ldots\right\}$. Fix $k \geq 0$ and given a labelled forest $F$ (which means we know the two sets $\operatorname{Prop}(F)$ and $\operatorname{Improp}(F)$ of $F$ ), a proper $k$-coloring of $F$ is a way of coloring all the internal vertices of $F$ so that for any $v \in I(F)$, if $v$ is proper then it can be colored by any color in $\left\{c_{1}, c_{2}, \ldots, c_{d_{v}}\right\}$; otherwise it can be colored by any color in $\left\{c_{1}, c_{2}, \ldots, c_{d_{v}}\right\} \cup$ $\left\{c_{1}^{\prime}, c_{2}^{\prime}, \ldots, c_{k}^{\prime}\right\}$. (Note that $c_{1}, c_{2}, \ldots c_{d_{v}}$ can be considered corresponding to the $d_{v}$ edges of $v$, and the colors $c_{1}^{\prime}, c_{2}^{\prime}, \ldots, c_{k}^{\prime}$ for improper vertices are considered as "special" colors.) Therefore, given a labelled forest $F$, there are $\prod_{v \in \operatorname{Prop}(F)} d_{v}$ $\prod_{v \in \operatorname{Improp}(F)}\left(d_{v}+k\right)$ proper $k$-colorings.

A $k$-colored labelled forest is a labelled forest with a proper $k$-coloring. Given a degree sequence $\mathbf{r}$ and $k \geq 0$, let $C \mathcal{F}_{\mathbf{r}, k}$ be the set of all $k$-colored labelled forests $F$ of type $\mathbf{r}$.

Lemma 3.1. $\mathcal{C} \mathcal{F}_{\mathbf{r}, k}$ is counted by $\sum_{F \in \mathscr{F}(\mathbf{r})} n ! \prod_{v \in I(F)}\left(\left(d_{v}+k\right)-\frac{k}{h_{v}}\right)$.

Proof. For any $F \in \mathscr{F}(\mathbf{r})$,

$$
\begin{aligned}
n ! \prod_{v \in I(F)}\left(\left(d_{v}+k\right)-\frac{k}{h_{v}}\right) & =n ! \sum_{J \subset I(F)}\left(\prod_{v \in J}-\frac{k}{h_{v}}\right)\left(\prod_{v \in I(F) \backslash J}\left(d_{v}+k\right)\right) \\
& =\sum_{J \subset I(F)} \frac{n !}{\prod_{v \in J} h_{v}}\left(\prod_{v \in J}-k\right)\left(\prod_{v \in I(F) \backslash J}\left(d_{v}+k\right)\right)
\end{aligned}
$$

However, $n ! / \prod_{v \in J} h_{v}$ is the number of labellings of $F$ so that all the vertices in $\mathrm{J}$ are 
proper. Therefore,

$$
\begin{aligned}
n ! & \prod_{v \in I(F)}\left(\left(d_{v}+k\right)-\frac{k}{h_{v}}\right) \\
= & \sum_{F^{\prime}} \sum_{J \subset \operatorname{Prop}\left(F^{\prime}\right)}\left(\prod_{v \in J}-k\right)\left(\prod_{v \in I(F) \backslash J}\left(d_{v}+k\right)\right)\left(F^{\prime} \text { is } F \text { with a labelling }\right) \\
= & \sum_{F^{\prime}} \sum_{J \subset \operatorname{Prop}\left(F^{\prime}\right)}\left(\prod_{v \in J}-k\right)\left(\prod_{v \in \operatorname{Prop}\left(F^{\prime}\right) \backslash J}\left(d_{v}+k\right)\right)\left(\prod_{v \in \operatorname{Improp}\left(F^{\prime}\right)}\left(d_{v}+k\right)\right) \\
= & \sum_{F^{\prime}}\left(\prod_{v \in \operatorname{Prop}\left(F^{\prime}\right)}\left(-k+d_{v}+k\right)\right)\left(\prod_{v \in \operatorname{Improp}\left(F^{\prime}\right)}\left(d_{v}+k\right)\right) \\
= & \sum_{F^{\prime}}\left(\text { the number of proper } k \text {-colorings } F^{\prime} \text { has }\right) .
\end{aligned}
$$

Summing over all of the forests in $\mathscr{F}(\mathbf{r})$ gives us

$$
\left|\mathcal{C F}_{\mathbf{r}, k}\right|=\sum_{F \in \mathscr{F}(\mathbf{r})} n ! \prod_{v \in I(F)}\left(\left(d_{v}+k\right)-\frac{k}{h_{v}}\right) .
$$

Now we look back at our hook length polynomials. We change (2.2) into another form which is more closely related to Postnikov's identity.

Lemma 3.2. Identity (2.2) has the following equivalent form:

$$
\sum_{F \in \mathscr{F}(\mathbf{r})} \prod_{v \in I(F)} \frac{\left(d_{v}+x\right) h_{v}-x}{d_{v} h_{v}}=\frac{\ell}{r_{1} ! r_{2} ! \cdots} \prod_{i=1}^{n-1}\left(r_{0}+i(1+x)\right) .
$$

Note that when $\mathbf{r}=(n+1,0, n, 0,0, \ldots)$ and $x=-1,(3.2)$ is the same as (1.1). Proof.

$$
\begin{aligned}
(2.2) & \Leftrightarrow \sum_{F \in \mathscr{F}(\mathbf{r})} \prod_{v \in I(F)} \frac{\left(\left(d_{v}-1\right) h_{v}+1\right) x+1-h_{v}}{d_{v} h_{v}}=\frac{\ell x}{r_{1} ! r_{2} ! \cdots} \prod_{i=1}^{n-1}\left(r_{0} x-i\right) \\
& \Leftrightarrow \sum_{F \in \mathscr{F}(\mathbf{r})} \prod_{v \in I(F)} \frac{\left(\left(d_{v}-1\right) h_{v}+1\right) x+\left(1-h_{v}\right) y}{d_{v} h_{v}}=\frac{\ell x}{r_{1} ! r_{2} ! \cdots} \prod_{i=1}^{n-1}\left(r_{0} x-i y\right) \\
& \Leftrightarrow \sum_{F \in \mathscr{F}(\mathbf{r})} \prod_{v \in I(F)} \frac{d_{v} h_{v} x+\left(1-h_{v}\right)(x+y)}{d_{v} h_{v}}=\frac{\ell x}{r_{1} ! r_{2} ! \cdots} \prod_{i=1}^{n-1}\left(r_{0} x-i y\right) \\
& \Leftrightarrow \sum_{F \in \mathscr{F}(\mathbf{r})} \prod_{v \in I(F)} \frac{\ell y}{d_{v} h_{v} y-\left(1-h_{v}\right) x}=\frac{\ell y}{r_{1} ! r_{2} ! \cdots} \prod_{i=1}^{n-1}\left(r_{0} y+i(x+y)\right) \\
& \Leftrightarrow(3.2)
\end{aligned}
$$


If we replace $x$ with $k$ in (3.2) and rearrange it a bit, we have

$$
\sum_{F \in \mathscr{F}(\mathbf{r})} n ! \prod_{v \in I(F)}\left(\left(d_{v}+k\right)-\frac{k}{h_{v}}\right)=\left(\begin{array}{c}
n \\
r_{1}, r_{2}, \ldots
\end{array}\right) \ell 1^{r_{1}} 2^{r_{2}} \cdots \prod_{i=1}^{n-1}\left(r_{0}+i(1+k)\right) .
$$

Comparing (3.1) and (3.3), we get the following proposition.

\section{Proposition 3.3.}

$$
\left|\mathcal{C F}_{\mathbf{r}, k}\right|=\left(\begin{array}{c}
n \\
r_{1}, r_{2}, \ldots
\end{array}\right) \ell 1^{r_{1}} 2^{r_{2}} \ldots \prod_{i=1}^{n-1}\left(r_{0}+i(1+k)\right) .
$$

However, we have a stronger result than Proposition 3.3. For any degree sequence $\mathbf{r}$, we denote by $V_{\mathbf{r}}$ the set of all ordered partitions $\mathbf{S}=\left(S_{1}, S_{2}, \ldots\right)$ of $[n]$ such that $\left|S_{i}\right|=r_{i}$. We let $\mathbf{S}=\left(S_{1}, S_{2}, \ldots\right)$ and $\mathcal{C} \mathcal{F}_{\mathbf{r}, k, \mathbf{S}}$ be the set of all the forests $F$ in $\mathcal{C} \mathcal{F}_{\mathbf{r}, k}$ such that $\forall v \in I(F)$, the label of $v$ is in $S_{d_{v}}$, that is, the label $i$ can only be used to label an internal vertex $v$ of degree $d$ if $i \in S_{d}$.

We call two partitions $\mathbf{S}^{(1)}$ and $\mathbf{S}^{(2)}$ adjacent if there exists $i \in[n-1]$, such that we can obtain $\mathbf{S}^{(1)}$ by swapping $i$ and $i+1$ in $\mathbf{S}^{(2)}$.

We construct a graph $G_{\mathbf{r}}$ with vertex set $V_{\mathbf{r}}$ and $\left\{\mathbf{S}^{(1)}, \mathbf{S}^{(2)}\right\}$ forming an edge in $G_{\mathbf{r}}$ if and only if they are adjacent. It's not hard to see that $G_{\mathbf{r}}$ is connected.

Lemma 3.4. For any two partitions $\mathbf{S}^{(1)}$ and $\mathbf{S}^{(2)}$ in $V_{\mathbf{r}}$,

$$
\left|\mathcal{C F}_{\mathbf{r}, k, \mathbf{S}^{(1)}}\right|=\left|\mathcal{C} \mathcal{F}_{\mathbf{r}, k, \mathbf{S}^{(2)}}\right| .
$$

Proof. It's enough to prove the case when $\mathbf{S}^{(1)}$ and $\mathbf{S}^{(2)}$ are adjacent.

Suppose that we obtain $\mathbf{S}^{(1)}$ by swapping $i$ and $i+1$ in $\mathbf{S}^{(2)}$, for some $i \in[n-1]$, and $i \in S_{d_{1}}^{(1)}, i+1 \in S_{d_{2}}^{(1)}$. (So $i \in S_{d_{2}}^{(2)}, i+1 \in S_{d_{1}}^{(2)}$.)

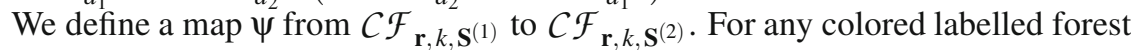
$F \in C \mathcal{F}_{\mathbf{r}, k, \mathbf{S}^{(1)}}$, let $v_{1}$ be the vertex with label $i$ and $v_{2}$ be the vertex with label $i+1$ :

(i) If $v_{1} \notin \operatorname{Des}\left(v_{2}\right)$ and $v_{2} \notin \operatorname{Des}\left(v_{1}\right)$, then $\psi(F)=(i, i+1) F$, where $(i, i+1) F$ means swap the labels $i$ and $i+1$.

(ii) If $v_{2} \in \operatorname{Des}\left(v_{1}\right)$ and $v_{1}$ is improper, then let $\psi(F)=(i, i+1) F$.

(iii) If $v_{2} \in \operatorname{Des}\left(v_{1}\right)$ and $v_{1}$ is proper (so $v_{2}$ is proper too), then let $\psi(F)=(i, i+1) F$.

(iv) If $v_{1} \in \operatorname{Des}\left(v_{2}\right)$ and $\exists j<i$ such that the vertex with label $j$ is in $\operatorname{Des}\left(v_{2}\right)$, then let $\psi(F)=(i, i+1) F$.

(v) If $v_{1} \in \operatorname{Des}\left(v_{2}\right)$, any vertex with label $j<i$ is not in $\operatorname{Des}\left(v_{2}\right)$, and color of $v_{2}$ is not one of the $k$ special colors, then let $\psi(F)=(i, i+1) F$.

(vi) If $v_{1} \in \operatorname{Des}\left(v_{2}\right)$, any vertex with label $j<i$ is not in $\operatorname{Des}\left(v_{2}\right)$ (so $v_{1}$ is proper and has a color $c_{\alpha}$ ), and the color of $v_{2}$ is one of the $k$ special colors, then we obtain $\psi(F)$ in the following way: Suppose that $v_{1}$ and $v_{2}$ are in tree $T$ with root $r$. Let $u$ be the $\beta$ th child of $v_{2}$ that is an ancestor of $v_{1}$ and $w$ be the $\alpha$ th child of $v_{1}$ that corresponds to the color of $v_{1}$. We separate $T$ at $v_{2}, u, v_{1}$, and $w$ to get five trees $T_{1}, T_{2}, T_{3}, T_{4}$, and $T_{5}$ with roots $r, v_{2}, u, v_{1}$, and $w$, respectively, denote by 
$v_{2}^{\prime}, u^{\prime}, v_{1}^{\prime}$, and $w^{\prime}$ the leaves of $T_{1}, T_{2}, T_{3}$, and $T_{4}$ obtained from $v_{2}, u, v_{1}$, and $w$, respectively. Attach the root $w$ of $T_{5}$ to $u^{\prime}$, attach the root $v_{2}$ of $T_{2}$ to $v_{1}^{\prime}$, attach the root $u$ of $T_{3}$ to $w^{\prime}$, and attach the root $v_{1}$ of $T_{4}$ to $v_{2}^{\prime}$. Color $v_{2}$ with the color $c_{\beta}$ corresponding to $u$ and $v_{1}$ with the original color for $v_{2}$. Finally, swap labels $i$ and $i+1$.

One can check that $\psi$ gives a bijection between $\mathcal{C} \mathcal{F}_{\mathbf{r}, k, \mathbf{S}^{(1)}}$ and $\mathcal{C} \mathcal{F}_{\mathbf{r}, k, \mathbf{S}^{(2)}}$.

We observe that Proposition 3.3 and Lemma 3.4 together are equivalent to the following Theorem:

\section{Theorem 3.5.}

$$
\left|\mathcal{C} \mathcal{F}_{\mathbf{r}, k, \mathbf{S}}\right|=\ell 1^{r_{1}} 2^{r_{2}} \cdots \prod_{i=1}^{n-1}\left(r_{0}+i(1+k)\right) .
$$

We also provide another proof of Theorem 3.5, which is bijective and combinatorial.

Proof. Let $C \mathcal{F}_{\mathbf{r}, k, \mathbf{S}, 1} \subset C \mathcal{F}_{\mathbf{r}, k, \mathbf{S}}$ be the set with all the forests with label 1 appearing in the first tree. Clearly, (3.4) is equivalent to

$$
\left|\mathcal{C F}_{\mathbf{r}, k, \mathbf{S}, 1}\right|=1^{r_{1}} 2^{r_{2}} \ldots \prod_{i=1}^{n-1}\left(r_{0}+i(1+k)\right) .
$$

Let $g_{i} \in\left[d_{i}\right], \forall 1 \leq i \leq n, f_{j} \in\left[r_{0}+j(1+k)\right], \forall 1 \leq j \leq n-1$. Then there are $1^{r_{1}} 2^{r_{2}} \cdots \prod_{i=1}^{n-1}\left(r_{0}+i(1+k)\right)$ choices for the $g_{i}$ 's and $f_{j}$ 's.

We will construct a bijection between $\left\{g_{i}, f_{j}\right\}$ and $\mathcal{C} \mathcal{F}_{\mathbf{r}, k, \mathbf{S}, 1}$ inductively on $n=$ $\sum_{d \geq 1} r_{d}$, the number of internal vertices of the forests.

When $n=1$, we have only one vertex. Suppose it has degree $d$. Then $r_{0}=$ $d+\ell-1$. We don't have $f_{j}$ 's and $g_{1} \in[d]$. Clearly, there's a natural bijection between the value of $g_{1}$ and the color of vertex 1 in any forest in $C \mathcal{F}_{\mathbf{r}, k, \mathbf{S}, 1}$.

Now we assume for $n<n_{0}$, we have a bijection between $\left\{g_{i}, f_{j}\right\}$ and $C \mathcal{F}_{\mathbf{r}, k, \mathbf{S}, 1}$, and consider $n=n_{0}$. For any $F \in C \mathcal{F}_{\mathbf{r}, k, \mathbf{S}, 1}$, let $T$ be it's first tree. We know that 1 is in $T$. We have two cases:

If the root of $T$ is 1 , then let $g_{1}$ be the value corresponding to the color of 1 . By removing 1 from $T, F$ becomes a forest with $\ell+d_{1}-1$ trees and $n-1$ vertices. However, the smallest vertex 2 is not necessarily in the first tree. Let $f_{n-1}$ be the position number of the tree containing 2 ; then $f_{n-1} \in\left[\ell+d_{1}-1\right]$. We cyclicly rotate the order of the trees so that that tree becomes the first tree in the forest and call the resulting forest $F^{\prime}$. Hence, $F^{\prime} \in \mathcal{C} \mathcal{F}_{\mathbf{r}^{\prime}, k, \mathbf{S}^{\prime}, 1}$, where $\mathbf{r}^{\prime}$ is obtained by subtracting 1 from $r_{1}$ in $\mathbf{r}$, and $\mathbf{S}^{\prime}$ is obtained from $\mathbf{S}$ by removing 1 from $S_{d_{1}}$. By the induction hypothesis, we can associate $\left\{g_{i}, f_{j}\right\}_{2 \leq i \leq n, 1 \leq j \leq n-2}$ to $F^{\prime}$. Including $g_{1}$ and $f_{n-1}$, we obtain a bijection between forests of this type and the set $\left\{g_{i}, f_{j}\right\}$ with $f_{n-1} \in$ $\left[\ell+d_{1}-1\right]$.

If the root of $T$ is $i(i \neq 1)$, then $i$ is improper and it can have $d_{i}+k$ choices of colors. We can associate these $d_{i}+k$ colors with choosing $f_{n-1}$ in the interval $\left[\ell+d_{1}+\sum_{j=2}^{i-1}\left(d_{j}+k\right), \ell+d_{1}-1+\sum_{j=2}^{i}\left(d_{j}+k\right)\right]$. Because 1 is in $T$, it is a descendant of $i$. Let $g_{i}$ be the number corresponding to the child of $i$ that is ancestor 
of 1 . Similar to the first case, remove $i$ and rotate the first $d_{i}$ trees so that 1 becomes contained in the first tree in the new forest $F^{\prime}$. Then $F^{\prime} \in \mathcal{C} \mathcal{F}_{\mathbf{r}^{\prime}, k, \mathbf{S}^{\prime}, 1}$, where $\mathbf{r}^{\prime}$ is obtained by subtracting 1 from $r_{i}$ in $\mathbf{r}$, and $\mathbf{S}^{\prime}$ is obtained from $\mathbf{S}$ by removing $i$ from $S_{d_{i}}$. Again, by a similar argument, we can get a bijection between forests of this type and the set $\left\{g_{i}, f_{j}\right\}$ with $f_{n-1} \in\left[\ell+d_{1}+\sum_{j=2}^{i-1}\left(d_{j}+k\right), \ell+d_{1}-1+\sum_{j=2}^{i}\left(d_{j}+k\right)\right]$.

Note that $\ell+d_{1}-1+\sum_{j=2}^{n}\left(d_{j}+k\right)=\ell+\sum_{d>1}(d-1) r_{d}+(n-1)(1+k)=r_{0}+$ $(n-1)(1+k)$. Therefore, we have constructed a bijection for $n=n_{0}$.

Remark 3.6. Because Theorem 3.5 implies Proposition 3.3, Proposition 3.3 together with Lemma 3.1 imply (3.2), and (3.2) is equivalent to (2.2), the above proof of Theorem 3.5 can be considered as another proof of Theorem 2.3. It also gives a new proof of (2.1) by substituting -1 for $x$ in (2.2) or substituting 0 for $x$ in (3.2).

Remark 3.7. One can modify our definition of $k$-colorings of a labelled forest so that it makes sense for $k=-1$. The proof of Theorem 3.5 works as well for $k=-1$.

\section{References}

1. Chen, W.Y.C.: A general bijective algorithm for trees. Proc. Natl. Acad. Sci. 87, 96359639 (1990)

2. Chen, W.Y.C., Yang, L.L.M.: On the hook length formula for binary trees. European J. Combin. 29, 1563-1565 (2008)

3. Du, R.R.X., Liu, F.: $(k, m)$-Catalan numbers and hook length polynomails for plane trees. European J. Combin. 28, 1312-1321 (2007)

4. Erdélyi, A., Etherington, I.M.H.: Some problems of non-associative combinations (2). Edinburgh Math. Notes 32, 7-12 (1940)

5. Gessel, I.M., Seo, S.: A refinement of Cayley's formula for trees. Electron. J. Combin. 11, \#R27 (2006)

6. Harary, H., Prins, G., Tutte, W.: The number of plane trees. Indag. Math. 26, 319-329 (1964)

7. Postnikov, A.: Permutohedra, associahedra, and beyond, Retrospective in Combinatorics: Honoring Richard Stanleys 60th Birthday, Massachusetts Institute of Technology, Cambridge (2004)

8. Postnikov, A.: Permutohedra, associahedra, and beyond. Int. Math. Res. Not. IMRN 2009, 1026-1106 (2009)

9. Seo, S.: A combinatorial proof of Postnikov's identity and a generalized enumeration of labeled trees. Electron. J. Combin. 11, \#N3 (2005)

10. R.P. Stanley, Enumerative Combinatorics, Vol. 2, Cambridge University Press, Cambridge, 1999.

Open Access This article is distributed under the terms of the Creative Commons Attribution Noncommercial License which permits any noncommercial use, distribution, and reproduction in any medium, provided the original author(s) and source are credited. 